dead, brain-dead patient, but then answers it himself correctly (ie, the patient's body). His objection is to our mention of the possibility of extending, perhaps indefinitely, the period during which the brain-dead patient might be maintained, which Lamb condemns as 'This confusion between medical facts and science fiction'.

In reply it will suffice to quote the lines which followed this mention in our paper, which incidentally were addressed to an argument of Lamb's:

'It is difficult to see why the brevity of the interval [between brain death and functional disintegration] should have any bearing on the definition of death. There are a host of medical conditions which, given the current power of medicine, also inevitably lead to the death of the system as a whole, just as renal failure did only a few years ago. There was and is no temptation to regard the onset of these conditions as the occasion of death, and, failing further argument, this judgment extends naturally to the brain.'

Lamb's remarks notwithstanding, the case for whole-brain death has not been successfully made. The tolerance of $\log$ ical disorder by the medical community in this matter is quite remarkable.

DANIEL WIKLER Associate Professor, University of Wisconsin Center for Health Sciences, Program in Medical Ethics, 470 North Charter Street, Madison, Wisconsin

\section{Reply to Professor Wikler}

SIR

Professor Wikler's reply to my review of his paper in Medicine and Moral Philosophy deserves careful attention. Not only does it call into question the concept of 'whole-brain death' advocated in the Report of the President's Commission for the Study of Ethical Problems in Medicine, there is also implicit dissatisfaction with the 'brain stem' concept of death which was advocated by the Royal Colleges of Medicine and their faculties in 1979 and more recently articulated in Dr C Pallis's The $A B C$ of Brain Stem Death in 1983 (1). In this reply I can do little more than indicate the substantive differences between Professor Wikler's position and my own, and invite further discussion on this extremely important issue.

Confusion in the brain death debate is often generated out of a failure to articulate the concept of death in question. The concept of death underlying my criticism of the original paper by Professor Wikler was 'the irreversible loss of integrated functioning of the organism as a whole'. This includes the irreversible loss of the capacity for consciousness and respiration. The point in the process of dying when criteria for this concept are met, is the moment when tests clearly indicate the death of the brain stem. The assumption underpinning this concept is that the brain stem is uniquely essential and, by the standards of present medical knowledge, irreplaceable.

In contrast, Professor Wikler advocates a "conception of death involving cognitive function'. If I understand this correctly he is saying that death, according to his conception, could be pronounced before tests had indicated an irreversible cessation of brain stem function. Yet part of his case for demonstrating the extent to which 'the whole brain concept is terminally [terminologically?] confused' rests on an appeal to methods of artificially maintaining certain integrative functions after brain stem death. But even according to a concept of death equated with loss of 'cognitive function' a patient without a functioning brain stem would be dead, since a functioning brain stem is essential to the capacity for consciousness and cognition. It is interesting that many philosophers seem unaware of the relation of the brain stem (and in particular of its reticular formation) to the capacity for consciousness.

Another part of Wikler's case against 'whole-brain' death consists of stressing the persistence of certain functions after brain stem death. Quite clearly this is a long way from his earlier emphasis on the 'loss of cognitive function'.

This argument seems to rest on a concept of 'bodily death', according to which as long as some organs continue to function after brain stem death the patient is deemed to be alive. From the standpoint of 'brain stem death' this concept is unacceptable. Brain stem death must be seen as the equivalent of decapitation (2). In such a state any residual functions, such as the temporary persistence of cardiac contractions, should not be regarded as life indicators. But on Wikler's terms if, following decapitation, exsanguination was prevented and ventilation and blood temperature were somehow maintained, the 'victim' could be said to be alive. It is doubtful whether any medical or legal community would endorse such a concept of death and it would be interesting to see if philosophical arguments could be produced to establish that a headless corpse was alive.

In cases involving the completion of embryological development after maternal brain stem death, which are hinted at by Wikler, I would suggest that there is no more evidence of maternal 'life' in the uterus of the ex-patient than in an incubator, or other artifact employed for fetal survival.

The substantive difference between us is that whereas Wikler sees brain stem death as a state of no greater significance than other medical conditions which lead to the death of the system as a whole, the brain stem conception of death, which I endorse, appeals to the fundamental uniqueness of the brain stem. Thus the difference I see between a patient ventilated after brain stem death and any other 'well-maintained occupant of an ICU' is not merely that the former will not recover, but that what is being ventilated is no longer an integrated organism surviving with the aid of mechanical support, but a combination of artificial procedures prolonging cellular life in certain cell aggregates. This is not to deny that an artifact, under other circumstances, can help to restore the organism to a state where it can function as an integrated whole. Kidney dialysis, mechanical pacemakers, and so on, can help restore the integration of the whole just as they can replace the loss of a part. But they do so primarily because they help to restore and/or maintain brain stem function. For this reason Wikler's derogation of the role of the brain stem to that of the kidney is unwarranted. Loss of renal function was lethal because it set off a chain of events which culminated in brain stem death. Machines can now prevent loss of brain stem function in these conditions and insofar as the kidney is replaceable the machine has eliminated a particular cause of brain stem death. But the brain stem itself remains irreplaceable. Science fiction begins when the critical role of the reticular formation is challenged.

\section{References}

(1) Pallis C. The ABC of brain stem death. London: British Medical Association, 1983.

(2) Pallis C. Brain stem death: the evolution of concept. In Morris P J, ed. Kidney transplantation. Second edition. New York, London and Orlando: Grune and Stratton, 1984.

DAVID LAMB

Department of Philosophy, University of Manchester 